\title{
Predicting the failure of thin porous membranes in bulge tests
}

\author{
Steven R. Gillmer ${ }^{\mathrm{a}, \mathrm{b}}$, David Z. Fang ${ }^{\mathrm{c}, \dagger}$, Sarah E. Wayson ${ }^{\mathrm{d}}$, Joshua D. Winans ${ }^{\mathrm{d}}$, \\ Niaz Abdolrahim ${ }^{\mathrm{b}, \mathrm{e}}$, Jon-Paul S. DesOrmeaux ${ }^{\mathrm{f}}$, Jirachai Getpreecharsawas ${ }^{\mathrm{d}, \mathrm{g}}$, \\ Jonathan D. Ellis ${ }^{\mathrm{b}, \mathrm{h}}$, Philippe M. Fauchet ${ }^{\mathrm{c}, \ddagger}$, James L. McGrath ${ }^{\mathrm{d}, *}$
}

${ }^{a}$ Lincoln Laboratory, Massachusetts Institute of Technology; Lexington, MA, USA

${ }^{\mathrm{b}}$ Department of Mechanical Engineering, University of Rochester; Rochester, NY, USA

${ }^{\mathrm{c}}$ Department of Electrical and Computer Engineering, University of Rochester; Rochester, NY, USA

${ }^{\mathrm{d}}$ Department of Biomedical Engineering, University of Rochester; Rochester, NY, USA

eMaterials Science Program, University of Rochester; Rochester, NY, USA

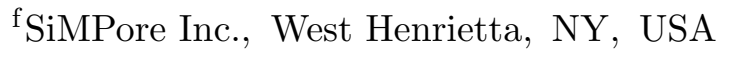

gMicrosystems Engineering, Rochester Institute of Technology; Rochester, NY, USA

${ }^{h}$ The Institute of Optics, University of Rochester; Rochester, NY, USA

${ }^{\dagger}$ Current address : Synapse Product Development, Inc. San Francisco, CA, USA

${ }^{\ddagger}$ Current address : Department of Electrical Engineering and Computer Science, Vanderbilt University, Nashville, TN, USA

${ }^{*}$ Corresponding author. Tel. + 1(585)273-5489; e-mail address: jmcgrath@bme.rochester.edu

(C) 2017. This manuscript version is made available under the Elsevier user license http://www.elsevier.com/open-access/userlicense/1.0/ 


\begin{abstract}
Silicon nanomembranes are thin nanoporous films that are frequently used as separation tools for nanoparticles and biological materials. In such applications, increased differential pressure across the nanomembranes directly increases process throughput. Therefore, a predictive tool governing the macroscale failure of the porous thin films is fundamentally important in application areas where high differential pressures are desired. Although the deflections and stresses of the nanomembranes can be reliably predicted, a straightforward and prognostic failure model has yet to be outlined. In this publication, a brittle macroscale failure model is established and validated with experimental results. Theoretical agreement with experiments within $10 \%$ accuracy offers reliable failure predictions for square membrane dimensions from $60 \mu \mathrm{m}$ to $1.5 \mathrm{~mm}$ through over 100 trials. The methodology relies on an effective fracture toughness from previously published work that is incorporated through Griffith's law. These developments will be useful in the selection of nanomembranes for particular applications and will help guide the design of materials with improved strength. The model should also prove useful for high-volume, in-line processing and inspection of nanomembranes as their role becomes more prominent in industry.
\end{abstract}




\section{Introduction}

The increased ductility of nanocrystalline ceramics at room temperature [1] has enabled a variety of promising technologies. Specifically, thin nanocrystalline membranes have the ability to withstand relatively high differential pressures without undergoing permanent mechanical yield [2]. Porous nanocrystalline silicon (pnc-Si) and porous $\mathrm{SiN}_{\mathrm{x}}$ (nanoporous nitride or NPN) membranes have leveraged their strength to demonstrate size-selective protein separations [3], high-permeability cell culture and tissue engineering [4,5], low-voltage electroosmosis [6], chemical capacitive sensing [7], and a potential replacement for current hemodialysis devices with increased performance and compact size [8-10]. In most filtration applications, increased differential pressure across the nanomembranes decreases process time. For this reason, the establishment of a predictive failure model is essential to produce stronger and more effective nanomembranes. Currently, the fabrication and control of pore properties is understood and documented $[8,11,12]$, stresses and deflections of the membranes are predictable [13-15]; however, a straightforward and conclusive failure mechanism has yet to be realized.

The most promising failure criteria has been published by Kovács, et al. [16, 17]. Their work accurately predicts the failure of ceramic membranes with micrometer-size pores with the incorporation of finite element analysis (FEA) and a required calibration factor to match experiments. Although the theory is reliable, it assumes the failure of the membranes occurs when the maximum principle stress in the material is attained. Indeed, this brittle failure mechanism can be considered valid; however, more common thin film failure analyses $[2,18,19]$ rely on the use of Griffith's law $[20,21]$ to elucidate a fracture toughness of the material without the need for FEA.

In this publication, we assimilate the tenable findings from Kovács, et al. $[16,17]$, Matoy, et al. [18], Di Maio and Roberts [19], and Yang, et al. [22] to predict the failure of thin and porous silicon-based membranes. An imperative finding of the finite element simulations performed by Kovács and the analytical explorations performed by Yang is that thin membranes produce maximum stress concentrations at the middle of the edges due to a large bending moment. Since considerable ambiguities in fracture toughness exist depending on the experimental setup [23], it is preferable to use values measured in a similar stress state to our current application. Conveniently, Matoy and Di Maio have published fracture toughness values of $\mathrm{SiN}_{\mathrm{x}}$ and monolithic silicon in a 
beam bending scenario. In this work, those fracture toughness values can be reliably applied to our predictive model for NPN and pnc-Si nanomembranes, respectively, since these materials fracture under a quasi-bending state near the middle of the edges.

The membranes investigated in this work are brittle; thus, their strength is governed by their largest flaw $[22,24]$. Figure 1 shows a scanning electron microscope image of a square membrane after fracture. Upon inspection of Figure 1a, it can be inferred that the largest flaw in these membranes is probably the pores. The fracture of the thin films is most likely initiated at these defects near the edges of the boundary where stresses are largest [15-17,22]. Furthermore, in Figure 1b, the fact that the membrane failed at all edges simultaneously is also consistent with the role of bending stresses at membrane edges causing failure. Our theory to predict these effects combines the principles of Timoshenko [13] with the appropriate weighting [15] to accurately predict stresses within the material under a bulge test scenario [14]. Under this criteria our hypothesis is that the pores in the material act as defects which can be related to failure using Griffith's Law $[20,21]$.

In comparison to the work by Kovács, et al., our approach does not require FEA for accuracy and can be used in a straightforward fashion. We note explicitly that this model is not meant to measure the fracture toughness of the thin films. Since it is well-known that fracture toughness can typically vary within $20 \%$, we slightly calibrate the values published by Matoy, et al. [18], and Di Maio and Roberts [19] within this bound for high accuracy.

\section{Construction of membranes}

Varying construction processes can yield different structural properties of the membranes. Methods for fabrication and control of pore properties in pnc-Si and NPN membranes have been published with extensive detail $[8,12]$. In this section, we briefly outline fabrication methods for completeness and accessible comparisons to our work. Figure 2 provides the process flow used to construct both types of nanoporous membranes used for this research. 


\section{Porous nanocrystalline silicon (pnc-Si)}

The approach to fabricate pnc-Si membranes utilizes silicon deposition techniques combined with etching processes. Directly patterning the pores is exceedingly time consuming and not fit to achieve industrial-scale throughput. Instead, the pores are spontaneously formed during a rapid thermal anneal whereby nanocrystals nucleate from amorphous silicon.

The first step in the process involves growing a $\sim 500 \mathrm{~nm}$-thick layer of $\mathrm{SiO}_{2}$ on both sides of a silicon substrate. The back side of the wafer is then patterned using photolithography to form an etch mask and the front oxide layer is removed and replaced with a three layer stack using RF magnetron sputtering. The pnc-Si membranes used in this research are $20 \mathrm{~nm}$ thick; thus, the

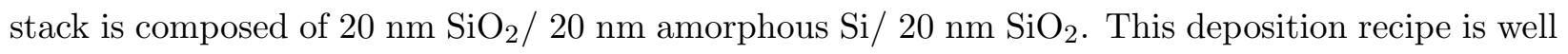
characterized, and can deposit films with $\pm 1 \%$ thickness accuracy and surface roughnesses under $0.5 \mathrm{~nm}[8]$.

To form the pores in pnc-Si, the wafer is briefly exposed to a high temperature $\left(800-1100^{\circ} \mathrm{C}\right.$ for $30 \mathrm{~s}$ ) in a rapid thermal processing chamber. The backside of the wafer is then etched using EDP (ethylenediamine pyrocatechol) which removes material along the (111) plane of the Si substrate. As a final step, the wafer is exposed to a buffered oxide etchant to remove oxide layers - thereby exposing the freestanding pnc-Si membrane. The membranes then tend to grow a native oxide layer that stabilizes at approximately $\sim 1.5 \mathrm{~nm}$ over the course of one week after being exposed to ambient air.

\section{Nanoporous nitride (NPN)}

The process used to create freestanding pnc-Si membranes is adapted for use as a mask in the fabrication of NPN [12]. The first step involves forming a $40 \mathrm{~nm}$ pnc-Si layer using a $50 \mathrm{~nm} \operatorname{SiN}_{\mathrm{x}} /$ $40 \mathrm{~nm}$ amorphous $\mathrm{Si} / 20 \mathrm{~nm} \mathrm{SiO}_{2}$ stack on the front side of the wafer. The backside is also coated with $\mathrm{SiN}_{\mathrm{x}}$. The thickness of the amorphous silicon layer is maximized to enable a longer etch through the $\mathrm{SiN}_{\mathrm{x}}$ after pores form in pnc-Si. To create pores, the wafer is exposed to a rapid thermal process (RTP in Figure 2) as outlined previously.

The backside $\mathrm{SiN}_{\mathrm{x}}$ layer is patterned to create an etch mask and the frontside is exposed to a reactive ion etching (RIE) process which transfers the pnc-Si pores to the $\mathrm{SiN}_{\mathrm{x}}$ layer. Next, 
using tetraethyl orthosilicate (TEOS) as a precursor gas, a $100 \mathrm{~nm} \mathrm{SiO}_{2}$ layer is deposited on the front surface using plasma-enhanced chemical vapor deposition (PECVD). The $\mathrm{SiO}_{2}$ layer protects the NPN during the bulk silicon etch from the backside of the wafer. The final step removes the protective $\mathrm{SiO}_{2}$ layer to expose the freestanding NPN membrane.

A variety of pnc-Si and NPN nanomembranes were constructed from both materials in square geometries ranging from $0.06 \mathrm{~mm}$ to $1.75 \mathrm{~mm}$ on a side. They were then subjected to a bulge test [14] until failure occurred [12]. Our objective was to develop a predictive theory that anticipated the pressures at which the nanomembranes failed.

\section{Failure Mechanism}

In 1921, Alan Griffith published a seminal paper [20] relating the strength of brittle materials to the sizes of their inherent defects and flaws rather than the intrinsic strength of their atomic bonds. Since then a general description of his findings, known as Griffith's Law, has become a cornerstone of fracture mechanics [21]:

$$
K_{I c}=\sigma_{\text {total }} Y \sqrt{\pi a}
$$

Equation 3.1 relates the fracture toughness of a material, $K_{I c}$, to the internal stress , $\sigma_{\text {total }}$ (in our case a combination of bending, tension and residual stresses), a dimensionless crack geometry factor, $Y$, and half the width of the defect or crack, $a$. Our hypothesis in this work is that the introduction of pores into a ultrathin membrane geometry drives failure of the membranes because the pores act as defects. The results will rely on an effective fracture toughness, $K_{I c}^{\text {eff }}$, rather than $K_{I c}$ for two main reasons. First, the defect size, $2 a$, is an average pore size without a consistent geometry (see Figure 1a), and second, we define the crack geometry factor, $Y$ as 1.1 for usability of the model [25]. More accurate fracture toughness measurements require precise insights and measurements of the exact value of $Y$ based on defect geometry $[18,19]$.

The majority of the following discussion is focused on realizing a critical stress, $\sigma_{\text {total }}$, within the material to use in Griffith's Law. According to Timoshenko [13], the full three-dimensional expression of this problem involves the simultaneous solution of two nonlinear differential equations. 
The general solution to these equations is unknown. Therefore, approximations need to be made to arrive at $\sigma_{\text {total }}$. Two common approximations suggested by Timoshenko involve (1) considering only the two-dimensional case in which the nonlinear governing equations are greatly simplified and can be solved or (2) assuming the plate has no resistance to bending. The former can be assumed in the case of very thin plates (nanomembranes) which have deflections many times larger than their thickness. However, due to the fact that the membrane failure has been observed to occur in a quasi-bending state at the edges $[13,15,17]$, the assumption that the material has no resistance to bending cannot be fully satisfied. Furthermore, the two-dimensional assumption cannot be reliably applied to square membranes either.

To reconcile the gap between the above assumptions, van Rijn, et al. [15] have proposed the weighted superposition of the two-dimensional deflection and stress equations with the threedimensional deflections and stresses predicted by neglecting bending resistance - effectively combining assumptions (1) and (2) from above to fit our application. We further expand their theory to include the residual stress of our nanomembranes which plays a role in ultimate fracture toughness values. Our adaptation of their approach is described as follows.

\subsection{Theoretical Model}

To begin, we first consider a two-dimensional clamped membrane subjected to a uniform pressure, q. A diagram of one such membrane in a bulge test experimental setup is shown in Figure 3 and has length, $l$, thickness, $h$, and is subjected to a distributed tensile force, $S$, as the membrane deforms under an applied load. The deflection of the membrane, $w$, is a function of its horizontal position, $x$, and assumes clamped boundary conditions.

In the two dimensional case, a rectangular plate of this form follows the well-known differential equation

$$
D \frac{d^{4} w(x)}{d x^{4}}-S \frac{d^{2} w(x)}{d x^{2}}=q
$$

with $D$ equal to the resistance to bending or flexural rigidity

$$
D=\frac{E h^{3}}{12\left(1-\nu^{2}\right)},
$$


where $E$ is Young's Modulus and $\nu$ is Poisson's ratio. Since we have assumed the two-dimensional case, the general solution for deflection follows [13]

$$
w(x)=\frac{q l^{4}}{16 u^{3} D \tanh (u)}\left\{\frac{\cosh \left[u\left(1-\frac{2 x}{l}\right)\right]}{\cosh (u)}-1\right\}+\frac{q l^{2}(l-x) x}{8 u^{2} D} .
$$

Equation 3.4 is a function of the dimensionless parameter, $u$, that is defined by

$$
u^{6}=\frac{9\left(1-\nu^{2}\right)^{2} q^{2} l^{8}}{8 E^{2} h^{8}}
$$

for the case that $u \gg 1$ which is generally satisfied for large deflections in the membranes [15]. In this form it can be shown that the distributed tensile force is equal to $S=4 u^{2} D / l^{2}$ [13]. Following this formalism, Timoshenko shows that a constant tensile stress in this two-dimensional material can be written as

$$
\sigma_{t}=\frac{S}{h}=\frac{4 u^{2} D}{h l^{2}}=\frac{E u^{2}}{3\left(1-\nu^{2}\right)}\left(\frac{h}{l}\right)^{2}
$$

where $u$ is still defined in Equation 3.5 for large deflections. Furthermore, the bending stress at the edge of the membrane follows [13]

$$
\sigma_{b}=\frac{3 q}{2 u \tanh (u)}\left(\frac{l}{h}\right)^{2}
$$

Under the condition $u \gg 1$ the term tanh $(u)$ approaches unity, and thus Equation 3.7 becomes

$$
\sigma_{b, u \gg 1}=\frac{3 q}{2 u}\left(\frac{l}{h}\right)^{2}
$$

Equations 3.6 and 3.8 comprise the resulting stresses in the two-dimensional assumption (1) which we have outlined. The next step involves evaluating the solution to our problem under assumption (2) in which the flexural rigidity, $D$, is set equal to zero. In that case, Timoshenko has used the principles of virtual displacements to calculate the tensile stress at the middle of a three-dimensional square membrane clamped at all four edges as [13]

$$
\sigma_{m}=\frac{E}{1-\nu} 1.848 \frac{\omega_{0}^{2}}{l^{2}},
$$


where the central deflection, $\omega_{0}$, is given by

$$
\omega_{0}=0.318 l\left(\frac{q l}{E h}\right)^{(1 / 3)}
$$

As previously stated, the problem presented in this publication cannot be described completely by assumptions (1) or (2). Instead, following the example of van Rijn [15], we impose the weighted superposition between the two-dimensional and three-dimensional case:

$$
\sigma_{\text {combined }}=\sigma_{m}\left(1+\frac{\sigma_{b}}{\sigma_{t}}\right)
$$

The assumption is dependent on the ratio between $\sigma_{b}$ and $\sigma_{t}$ scaling identically at the edge of the membrane so that the ratio between them remains constant. Finally, we incorporate the residual stress in our membranes with a simple addition:

$$
\sigma_{\text {total }}=\sigma_{\text {combined }}+\sigma_{r}
$$

Equation 3.12 is combined with Equation 3.1 to produce a complete failure criteria for the membranes. The resulting equation is rearranged to form

$$
q_{f}=\sqrt{\left(\frac{K_{I c}^{\mathrm{eff}}}{Y \sqrt{\pi a} C_{\nu}}-\frac{\sigma_{r}}{C_{\nu}}\right)^{3} \frac{h^{2}}{l^{2} E}},
$$

where $C_{\nu}$ is a constant derived from Equations 3.6, 3.8 and 3.9 that depends on Poisson's ratio. For our membranes made out of NPN ( $\nu=0.28$ from Ref. [14]) and pnc-Si $(\nu=0.22$ from Ref. [26]) Equations 3.6, 3.8 and 3.9 reduce to

$$
\begin{aligned}
\sigma_{t, \mathrm{NPN}} & =0.356 \sqrt[3]{\frac{q^{2} l^{2} E}{h^{2}}} ; \quad \sigma_{b, u \gg 1, \mathrm{NPN}}=1.511 \sqrt[3]{\frac{q^{2} l^{2} E}{h^{2}}} ; \quad \sigma_{m, \mathrm{NPN}}=0.260 \sqrt[3]{\frac{q^{2} l^{2} E}{h^{2}}} \\
\sigma_{t, \mathrm{pnc}-\mathrm{Si}} & =0.352 \sqrt[3]{\frac{q^{2} l^{2} E}{h^{2}}} ; \quad \sigma_{b, u \gg 1, \mathrm{pnc}-\mathrm{Si}}=1.495 \sqrt[3]{\frac{q^{2} l^{2} E}{h^{2}}} ; \quad \sigma_{m, \mathrm{pnc}-\mathrm{Si}}=0.240 \sqrt[3]{\frac{q^{2} l^{2} E}{h^{2}}} .
\end{aligned}
$$

Thus the above expressions can be rearranged in the form of Equation 3.13 to predict the pressures, $q_{f, \mathrm{NPN}}$ and $q_{f, \mathrm{pnc}-\mathrm{Si}}$ at which the membranes will fail: 


$$
\begin{aligned}
q_{f, \mathrm{NPN}} & =\sqrt{\left(\frac{K_{I c}^{\mathrm{eff}}}{Y \sqrt{\pi a}(1.364)}-\frac{\sigma_{r}}{1.364}\right)^{3} \frac{h^{2}}{l^{2} E}} \\
q_{f, \mathrm{pnc}-\mathrm{Si}} & =\sqrt{\left(\frac{K_{I c}^{\mathrm{eff}}}{Y \sqrt{\pi a}(1.259)}-\frac{\sigma_{r}}{1.259}\right)^{3} \frac{h^{2}}{l^{2} E}} .
\end{aligned}
$$

We define $Y \approx 1.1$ which designates that failure will occur near the edge of the clamped membrane [25] where stress concentrations are highest.

We note that another commonly used approach for adding residual stress is through a root-sumsquare (RSS) approach. The linear superposition which we assume in Equation 3.12 is considered the worst-case scenario in which residual stress has the most significant role. Realistically, however, the actual weighting of residual stress in the material is probably somewhere in between the linear and RSS weighting. Further investigation is needed to reconcile what the exact weighting for these nanomembranes should be. For the present work, a highly accurate weighting of residual stress is not necessary since it has a near-negligible effect on burst pressure measurements. This will become evident in the experimental results section when we explore the effect of varying residual stress on burst pressure (see Figure 7c).

\section{Determination of Model Parameters}

Prior to burst pressure measurements, some model parameters need to be determined for the use of Equations 3.16 and 3.17. Those parameters include Young's modulus, E, residual stress, $\sigma_{r}$, sidewall length, $l$, and pore size, $2 a$. The thickness of the membranes, $h$, can be reliably predicted during the construction of the membranes and is not described in this section.

\subsection{Young's Modulus and Residual Stress}

Young's moduli and residual stresses of the porous membranes were directly measured using the bulge test technique [14]. The method requires a data fit using a least-squares regression algorithm with Young's modulus and residual stress as parameters under the formalism

$$
q=C_{1} \sigma_{r} h \frac{w_{0}}{(l / 2)^{2}}+C_{2} M h \frac{w_{0}^{3}}{(l / 2)^{4}}
$$


The $C_{1}$ and $C_{2}$ coefficients are dependent on the membrane geometry and the film's Poisson ratio, $\nu, q$ is the pressure applied to the membrane, $\sigma_{r}$ is the film's residual stress, $h$ is the thickness of the film, $w_{0}$ is the vertical displacement at the center of the membrane, $l$ is the length of the square window, and $M$ is the biaxial modulus, $E /(1-\nu)$, for an isotropic film. For a square membrane, Vlassak calculated $C_{1}=3.393$ and $C_{2}=(0.792+0.085 \nu)^{-3}$ using energy minimization techniques to match the free energy of the membrane with the membrane displacement field [14].

A Veeco Wyko NT1100 (Santa Clara, CA) white light interferometer was used to interrogate the change in membrane height under an applied pressure. The instrument uses a technique known as vertical-scanning interferometry to achieve a range of $2 \mathrm{~mm}$ and resolution of $3 \mathrm{~nm}$ during bulge tests. Figure 3 shows a schematic of the experimental setup. A membrane chip is sealed inside a custom made enclosure using an O-ring and tightened bolts. The chamber is pressurized with compressed Argon from the side that is sealed with the O-ring. A $0-689 \mathrm{kPag}(0-100 \mathrm{psig})$ gas regulator (Harris \#301-100-580) and manometer (VWR \#33500-084) are used to control the pressure. To confirm there were no gross leaks in the system, a solid silicon chip was sealed inside the enclosure and pressurized. The pressure decreased less than $3.4 \mathrm{kPa}$ over five minutes. Therefore, it can be assumed that there is sufficient accuracy in the pressure measurement over the duration of data acquisition (less than one minute). The interferometric objective is placed directly above the membrane window. Pressure is increased in $0.7 \mathrm{kPa}$ increments as the height data is recorded with sufficient time in between measurements to let transient flow settle into a steady state. The described setup was used in this fashion to record bulge test results (Figure 4) to determine material properties. The same setup was used to record burst pressure measurements (Figure 6).

Porosity typically affects the stiffness of the nanomembranes. Although it does not change material properties, the effects can be accounted for by scaling solid Young's modulus as $E_{p}=$ $(1-P) E_{s}$ where $P$ is the membrane porosity, $E_{p}$ is porous effective Young's modulus, and $E_{s}$ is the solid Young's modulus $[16,27]$. Likewise, residual stress can be scaled according to $\sigma_{r, p}=$ $(1-P) \sigma_{r, s}$. A notable departure from this model was observed by Kovács, et al. [17] which required additional exponential scaling of the porosity. In this work, we avoid all porosity scaling ambiguities by measuring the porous effective Young's moduli and effective residual stresses in situ via Equation 4.1; therefore, no scaling is required. Although we did not use the above scaling 
factors to measure the values in Table 1, those equations are used later to create Figure 7b.

Bulge test results are shown in Figure 4. The porous pnc-Si membrane in Figure 4 had a sidewall length of $67 \mu \mathrm{m}$ and the NPN membranes had sidewall lengths of $278 \mu \mathrm{m}$. An exploration of the model sensitivity in the experimental results section will demonstrate that it is absolutely critical to accurately measure sidewall lengths for small membrane geometries such as these. For reliable bulge test results on larger membranes, the requirement is not as stringent.

The fitted values for porous effective Young's moduli and porous effective residual stresses of the membranes have been measured using a single bulge test measurement trial (Figure 4). For measurements similar to ours with silicon-based membranes, previously published work has shown that Young's modulus and residual stress can be measured within $\sim 2 \%$ and $\sim 1 \%$ variation, respectively, over ten trials [28]. Therefore, we assume our measured values have sufficient accuracy.

\subsection{Sidewall Length}

To accurately measure sidewall lengths, the membranes were inspected under a light microscope (Olympus BX51). Using a camera on the light microscope, images were captured of the membranes and a scale artifact at the same magnification. The scale was then used to calibrate the images and measure the sidewall lengths in the image processing program, ImageJ. Although the substrate edges have angled faces from acid etching, the ambiguity of that effect on sidewall length (on the order of tens of nanometers) was negligible since the resolution of our sidewall length measurements was on the order of single micrometers.

\subsection{Pore Size}

Pnc-Si and NPN have a distinct advantage over other porous membranes of being amenable to characterization by transmission electron microscopy (TEM) due to small thickness. As such, pore distributions can be directly calculated from TEM micrographs using custom MATLAB scripts (Figure 5). During construction of the membranes, samples compatible with a TEM specimen holder were integrated on each wafer in several radial positions.

The MATLAB routine (available at nanomembranes.org/resources/software) inputs a digital TEM micrograph of pnc-Si or NPN. The first operations performed on an image involve removing 
"salt and pepper" or speckle noise using a median filter and a background correction algorithm, which uses a nearest neighbor averaging algorithm to smooth out any variations in the lighting due to non-uniformity in the electron beam. Next, a threshold value is specified to separate the pores (high intensity pixels) from background features (low intensity pixels). The result is a binary image where pores are highlighted in white (' 1 ') and the solid film is black (' 0 ') (Figure 5a). Frequently, the threshold procedure will yield false positives; that is, areas of solid film that are highlighted white. To remove these areas, an iterative process of pixel dilation, erosion, opening, and closing is performed [29]. Any remaining false positives are identified manually by comparing the binary image with the original micrograph and removed. Finally, pore statistic calculations are performed and pore sizes are binned into a histogram.

After acquiring a pore size distribution, the histogram was fit using a scaled Weibull probability density function [30] in the form of:

$$
y=c\left(\frac{x}{a}\right)^{b} \exp \left[-\left(\frac{x}{a}\right)^{b}\right]
$$

where $a$ and $c$ scale the horizontal and vertical dimensions and $b$ determines the shape of the curve (Figure 5c). Estimating the pore density as a continuous curve allows us to graphically compare sets of distributions on the same plot. It should be noted that due to the nature of pore formation in pnc-Si and NPN, a sharp pore size cut-off can be defined. Thus, an exponential fit like the Weibull function may be used to characterize the pore size distribution. In contrast, conventional ultrafiltration membranes have a much wider pore size distribution described by log-normal fits [31].

\section{Pore Size Distribution Error Analysis}

It is evident in Figure 5 that our membranes possess average pore diameters rather than well-defined cracks. For this reason, we only specify an "effective fracture toughness" in Equations 3.16 and 3.17 to predict when the membranes will burst. An accurate measurement of the actual fracture toughness of the materials requires well-defined defects and is subject to experimental variations [23]. The variations in pore diameter and shape also limit us from defining a more accurate geometry factor, $Y$, in Equations 3.16 and 3.17 . Therefore we approximate $Y \approx 1.1$ which is generally assumed as a first-order estimate when cracks at the edge of samples promote 
failure [25]. To maintain the usability of this model for high-volume inspection and processing of the nanomembranes, we neglect a meticulous search for the largest crack (or pore) on each sample, which would therefore yield a better estimate of $Y$. The reader may also pose the question of why we chose a value of 1.1 as opposed to simply 1 for $Y$. The difference between $Y=1.1$ and $Y=1$ in Equations 3.16 and 3.17 correlates to a nominal change in burst pressure of $15-16 \%$ for the membranes presented in this work. Therefore, more research is needed to justify a departure from the 1.1 value we currently assume since the value of $Y$ cannot be inconsequentially changed to 1. Despite these assumptions, it will be shown that our current value for $Y$ predicts effective fracture toughness values that agree well to previously published fracture toughness measurements that were investigated under more stringent conditions with scrupulous geometry factor analysis.

Variations in porosity over the entire wafer also needed to be investigated. Typically, a single TEM micrograph is used to characterize the pore size distribution across an entire wafer. However, there are inevitable errors associated with analysis using a low sample size. Pore statistics are generated from an $11 \mu \mathrm{m} \times 17 \mu \mathrm{m}$ micrograph. One natural question that should be raised is whether this area is representative of the entire membrane area, which spans tens of thousands of square micrometers. To investigate this, the average pore diameter and membrane porosity were calculated at 10 different points on a representative $200 \mu \mathrm{m}$ x $200 \mu \mathrm{m}$ pnc-Si membrane. The membrane used in that exploration was not used in the experimental results section of this publication. The median average pore size and membrane porosity were $19.6 \mathrm{~nm}$ and $6.9 \%$, respectively, with standard deviations in average pore diameter and membrane porosity of $0.1 \mathrm{~nm}$ and $0.2 \%$. From these results, it is concluded that there is very little variation in morphology within a single membrane window. Thus, statistical analysis of one region of interest will be sufficiently representative of the entire membrane area. Pore diameter and porosity have been rounded to the nearest integer for all results presented in this publication.

\section{Experimental Results}

To determine membrane burst pressures, bulge test experiments were conducted with increasing pressure until membrane failure. In these experiments, the top side of the membrane was always facing the applied pressure as shown in Figure 3. This orientation ensures delamination effects 
did not play a role in failure. Equations 3.16 and 3.17 were then incorporated into MATLAB to compare theory against experiment. Results are shown in Figure 6 and a set of parameters, including best fit estimates of $K_{I c}^{\mathrm{eff}}$, are found in Table 1 . The error bars on Figure 6 represent \pm one standard deviation. Standard deviations of the pnc-Si failure pressures are based on a minimum of 19 trials and standard deviations of all NPN failure results are based on a minimum of four trials. The agreement between experiment and theory in Figure 6 proves Griffith's Law is reliable for high-volume, in-line prediction of failure based on nominal parameters.

For $50 \mathrm{~nm}$ thick NPN films with $66 \mathrm{~nm}$ pores and $20 \%$ porosity, $K_{I c}^{\mathrm{eff}} \approx 1.45 \mathrm{MPa} \sqrt{\mathrm{m}}$; for $50 \mathrm{~nm}$ thick NPN films with $55 \mathrm{~nm}$ pores and $14 \%$ porosity, $K_{I c}^{\text {eff }} \approx 1.41 \mathrm{MPa} \sqrt{\mathrm{m}}$; and for $20 \mathrm{~nm}$ thick pnc-Si films with $28 \mathrm{~nm}$ pores and $7 \%$ porosity, $K_{I c}^{\mathrm{eff}} \approx 0.98 \mathrm{MPa} \sqrt{\mathrm{m}}$. These nominal values were initially retrieved from the work of Matoy, et al. [18], and Di Maio and Roberts [19]. After slight calibrations within the uncertainty limits of measured fracture toughness (generally 20\%), we have arrived at our current values. A direct comparison between our work and that of Matoy, Di Maio and other notable publications is shown in Table 2. Using these values for $K_{I c}^{\mathrm{eff}}$, our model can predict mean membrane failure pressures within $10 \%$ accuracy for square membrane dimensions from $60 \mu \mathrm{m}$ to $1.5 \mathrm{~mm}$.

Exploring the parameter space of the simulation offers insight into the sensitivity of the model and may identify design strategies for creating stronger membranes. Figure 7 shows predicted changes in the burst pressures of pnc-Si membranes as a function of fracture toughness, porosity, residual stress, pore size, membrane thickness, and error in side length measurement. The exploration shows the most significant changes in burst pressure occur with variations in fracture toughness (a), pore size (d), and membrane thickness (e), while changes in porosity (b) and residual stress (c) have minimal effects on the failure of the material. Inaccuracy in the measured side lengths of square membranes (f) are shown to have a much more significant effect on smaller membranes compared to larger ones. The main conclusion to be drawn from Figure $7 \mathrm{f}$ is that a slight error in the measurement of window lengths $(e . g . \approx 10 \mu \mathrm{m})$ could easily be the dominant source of error in burst pressure measurements. The burst pressure axis scale has been maintained across all plots for an intuitive comparison of parameter sensitivities.

A final remark should be made in regards to the deduction that higher porosities yield slightly 
higher strengths of the material (Figure 7b). Although mathematical porosity scalings were not required for the values in Table 1, the simulation in Figure $7 \mathrm{~b}$ assumes the relationships $E_{p}=$ $(1-P) E_{s}$ and $\sigma_{r, p}=(1-P) \sigma_{r, s}$ which decrease the effective Young's moduli and residual stresses with higher porosities. These scalings generally make the membrane more flexible and therefore more resistant to failure.

\section{Discussion and Conclusions}

We developed a mathematical model based on Griffith's law that predicts burst pressures of thin nanoporous membranes based on an effective fracture toughness from previously published literature. Our experiments show that porous nanocrystalline silicon and silicon nitride membranes fail under catastrophic brittle fracture. Assuming a macroscopic brittle behavior and considering the average size of the pores as the initial crack size, our model calculates the resultant bulge test pressure that membranes can withstand before fracture. The significant outcome of this work is a mathematical model for predicting burst pressure that accounts for the effect of various structural parameters on the macroscopic behavior of the nanomembranes including variations in thickness, pore size, and porosity. The model predictions are in agreement with experimental results within $10 \%$ accuracy for square membrane dimensions from $60 \mu \mathrm{m}$ to $1.5 \mathrm{~mm}$ through over 100 trials. The model is very straightforward and only needs to slightly calibrate one parameter - effective fracture toughness. Unlike the previous approaches $[16,17]$, our method does not require FEA for accuracy.

This publication deduces fracture toughness of porous NPN membranes to be much lower than their non-porous counterparts [2] in a bulge test scenario. Our mathematical model predicts a fracture toughness of $\sim 1.43 \mathrm{MPa} \sqrt{\mathrm{m}}$ for NPN membranes, while Merle and Göken [2] measured values around $6 \mathrm{MPa} \sqrt{\mathrm{m}}$ for solid non-porous membranes. Although this discrepancy may appear problematic, we attribute the disagreement to experimental conditions. It should be noted that large variations in nanoscale fracture toughness values occur as a consequence of different loading conditions [23]. Merle and Göken fabricated defects in the center of their membranes which promoted failure in pure tension. In contrast, our membranes' defects lie at the boundaries which catalyzes failure under a combination of bending and tensile stress. The combination of bending and tension produces larger stresses compared to pure tension for the same applied bulge test 
pressure. To reinforce this significance, Matoy, et al. [18] have measured the fracture toughness for $\mathrm{SiN}_{\mathrm{x}}$ micro-cantilevers to be approximately $1.2-1.4 \mathrm{MPa} \sqrt{\mathrm{m}}$ (see Table 2) when subjected to pure bending. The findings of Matoy, et al. not only validate our observations, they lead to the conclusion that bending is most likely the dominant failure mode in NPN nanomembranes. Our observed fracture toughness of pnc-Si nanomembranes $(\sim 0.98 \mathrm{MPa} \sqrt{\mathrm{m}})$ is validated by its agreement to that of bulk monocrystalline silicon [19,33-35].

Nanomembranes are now used in research and commercial settings for separations $[3,8,10]$, cell culture [4,5], pumping [6], and sensing [7]. The volumes of fluids that can be processed in these applications are limited by the size of membranes that can be reliably constructed. Stronger membranes are not only less susceptible to bulge test failure, they result in higher yields during manufacturing and fewer failures during device assembly. Therefore, if a reliable understanding of the mechanics of nanomembrane failure can enable larger area devices, it can both lower cost and increase utility. The model developed here not only provides an excellent fit to the burst pressure data, it helps identify which parameters should be targeted in manufacturing in order to improve nanomembrane mechanics.

Our work over the years has identified a nominal burst pressure value of $\sim 30 \mathrm{kPa}$ for reliable use of nanomembranes in many application areas. The experimental findings in this publication show that NPN crosses this threshold when membranes are smaller than $\sim 0.7 \mathrm{~mm}$ on a side while pnc-Si membranes require side lengths less than $0.25 \mathrm{~mm}$ to exceed this threshold. This finding is consistent with a significant increase in the number of devices and applications we have been able to explore since the introduction of NPN [12]. Since NPN and pnc-Si porosities, thicknesses, and elastic moduli are comparable, the primary explanation for the improved strength of NPN compared to pnc-Si appears to be the increase in fracture toughness. Ultimately we expect that atomistic simulations of nanomembranes will be the key to selecting materials and designing ultrastructures to maximize fracture toughness. More immediately, our sensitivity study identifies smaller pore sizes and increased membrane thickness as the parametric targets for improved membrane strength. Of course both of these parameters impact membrane performance as a molecular filter and thus any increases in strength must be weighed against performance goals. 


\section{Acknowledgments}

The authors greatfully acknowledge valuable discussions with Dr. John Lambropoulos and Dr. Michael Skarlinski (Materials Science Program, University of Rochester) as well as with Dr. Karan Mehrotra (Corning Incorporated) during the development of this research. This work was funded by the National Science Foundation under award CBET:1159579. Steven Gillmer is currently an MIT Lincoln Laboratory employee. No Laboratory funding or resources were used to produce the result/findings reported in this publication. Opinions, interpretations, conclusions, and recommendations are those of the authors, and do not necessarily represent the view of the United States Government. 


\section{References}

[1] J. Karch, R. Birringer, and H. Gleiter. Ceramics ductile at low temperature. Nature, 330:556$558,1987$.

[2] B. Merle and M. Göken. Fracture toughness of silicon nitride thin films of different thicknesses as measured by bulge tests. Acta Materialia, 59:1772-1779, 2011.

[3] Thomas R. Gaborski, Jessica L. Snyder, Christopher C. Striemer, David Z. Fang, Michael Hoffman, Philippe M. Fauchet, and James L. McGrath. High-performance separation of nanoparticles with ultrathin porous nanocrystalline silicon membranes. ACS Nano, 4(11):6973-6981, 2010.

[4] A.A. Agrawal, B.J. Nehilla, K.V. Reisig, T.R. Gaborski, D.Z. Fang, C.C. Striemer, P.M. Fauchet, and J.L. McGrath. Porous nanocrystalline silicon membranes as highly permeable and molecularly thin substrates for cell culture. Biomaterials, 31(20):5408-5417, 2010.

[5] Andrea R. Mazzocchi, Alan J. Man, Jon-Paul S. DesOrmeaux, and Thomas R. Gaborski. Porous membranes promote endothelial differentiation of adipose-derived stem cells and perivascular interactions. Cellular and Molecular Bioengineering, 7(3):369-378, 2014.

[6] Jessica L. Snyder, Jirachai Getpreecharsawas, David Z. Fang, Thomas R. Gaborski, Christopher C. Striemer, Philippe M. Fauchet, David A. Borkholder, and James L. McGrath. High-performance, low-voltage electroosmotic pumps with molecularly thin silicon nanomembranes. Proceedings of the National Academy of Sciences of the United States of America, 110(46):18425-18430, 2013.

[7] Maryna N. Kavalenka, Christopher C. Striemer, Jon-Paul S. DesOrmeaux, James L. McGrath, and Philippe M. Fauchet. Chemical capacitive sensing using ultrathin flexible nanoporous electrodes. Sensors and Actuators B: Chemical, 162(1):22-26, 2012.

[8] Christopher C. Striemer, Thomas R. Gaborski, James L. McGrath, and Philippe M. Fauchet. Charge- and size-based separation of macromolecules using ultrathin silicon membranes. Nature, 445:749-753, 2007. 
[9] Albert van den Berg and Matthias Wessling. Silicon for the perfect membrane. Nature, 445:726, 2007.

[10] Dean G. Johnson, Tejas S. Khire, Yekaterina L. Lyubarskaya, Karl J. P. Smith, JonPaul S. DesOrmeaux, Jeremy G. Taylor, Thomas R. Gaborski, Alexander A. Shestopalov, Christopher C. Striemer, and James L. McGrath. Ultrathin silicon membranes for wearable dialysis. Advances in Chronic Kidney Disease, 20(6):508-515, 2013.

[11] D.Z. Fang, C.C. Striemer, T.R. Gaborski, J.L. McGrath, and P.M. Fauchet. Methods for controlling the pore properties of ultra-thin nanocrystalline silicon membranes. Journal of Physics: Condensed Matter, 22(45), 2010.

[12] J.P.S. DesOrmeaux, J.D. Winans, S.E. Wayson, T.R. Gaborski, T.S. Khire, C.C. Striemer, and J.L. McGrath. Nanoporous silicon nitride membranes fabricated from porous nanocrystalline silicon templates. Nanoscale, 6:10798-10805, 2014.

[13] Stephen P. Timoshenko and S. Woinowsky-Krieger. Theory of plates and shells. McGraw-Hill Companies, 1959.

[14] J.J. Vlassak and W.D. Nix. A new bulge test technique for the determination of young's modulus and poisson's ratio of thin films. Journal of Materials Research, 7(12):3242-3249, 1992.

[15] Cees van Rijn, Michiel van der Wekken, Wietze Nijdam, and Miko Elwenspoek. Deflection and maximum load of microfiltration membrane sieves made with silicon micromachining. IEEE Journal of Microelectromechanical systems, 6(1):48-54, 1997.

[16] A. Kovács, Á. Kovács, M. Pogány, and U. Mescheder. Mechanical investigation of perforated and porous membranes for micro- and nanofilter applications. Sensors and Actuators B: Chemical, 127(1):120-125, 2007.

[17] Á. Kovács, A. Kovács, and U. Mescheder. Estimation of elasticity modulus and fracture strength of thin perforated sin membranes with finite element simulations. Computational Materials Science, 43:59-64, 2008. 
[18] Kurt Matoy, Helmut Schönherr, Thomas Detzel, Thomas Schöberl, Reinhard Pippan, Christian Motz, and Gerhard Dehm. A comparative micro-cantilever study of the mechanical behavior of silicon based passivation films. Thin Solid Films, 518:247-256, 2009.

[19] D. Di Maio and S.G. Roberts. Measuring fracture toughness of coatings using focused-ionbeam-machined microbeams. Journal of Materials Research, 20(2):299-302, 2005.

[20] A.A. Griffith. The phenomena of rupture and flow in solids. Philosophical Transactions of the Royal Society of London, 221:163-198, 1921.

[21] G.R. Irwin. Analysis of stresses and strains near the end of a crack traversing a plate. Journal of Applied Mechanics, pages 361-364, 1957.

[22] J. Yang, J. Gaspar, and O. Paul. Accuracy and reliability of bulge test experiments. Journal of Microelectromechanical Systems, 17(5):1120-1134, 2008.

[23] Taechung Yi and Chang-Jin Kim. Measurement of mechanical properties for MEMS materials. Measurement Science and Technology, 10(8):706-706, 1999.

[24] D.G.S. Davies. The statistical approach to engineering design in ceramics. Proc. Brit. Ceramic Soc., 22:429-452, 1973.

[25] Marc Meyers and Krishan Chawla. Mechanical Behavior of Materials: Second Edition. Cambridge University Press, Ch. 7, pg. 427, 2009.

[26] David Z. Fang. Fabrication, Characterization, and Functionalization of Porous Nanocrystalline Silicon Membranes. PhD thesis, University of Rochester, 2010.

[27] J. Harrop and R.M. Abdul-Karim. Stresses and deflections in circular plates with square pitch perforations. Nuclear Engineering and Design, 6(5):431-439, 1967.

[28] M.K. Small, J.J. Vlassak, S.F. Powell, B.J. Daniels, and W.D. Nix. Accuracy and reliability of bulge test experiments. Mat. Res. Soc. Symp. Proc., 308:159-164, 1993.

[29] R.V.D. Boomgaard and R.V. Balen. Methods for fast morphological image transforms using bitmapped binary images. CVGIP: Graph. Models Image Proc., 54:252-258, 1992. 
[30] J.F. Lawless. Statistical Models and Methods for Lifetime Data, 2nd ed. John Wiley Sons, Inc., 2002.

[31] A. Mehta and A.L. Zydney. Permeability and selectivity analysis for ultrafiltration membranes. J. Membrane Sci., 249:245-249, 2005.

[32] G.R. Anstis, P. Chantikul, B.R. Lawn, and D.B. Marshall. A critical evaluation of indentation techniques for measuring fracture toughness: I, direct crack measurements. Journal of the American Ceramic Society, 64(9):533-538.

[33] B. Wong and R.J. Holbrook. Microindentation for fracture and stress-corrosion cracking studies in single-crystal silicon. J. Electrochem. Soc.: Solid-State Science and Technology, 134(9):2254-2256, 1987.

[34] G. Michot and A. George. Fracture and crack tip plasticity in silicon and gallium arsenide. Inst. Phys. Conf. Ser. Chapter 4, (104):385-396, 1989.

[35] D. Picard, D. Leguillon, and C. Putot. A method to estimate the influence of the notch-root radius on the fracture toughness measurement of ceramics. Journal of the European Ceramic Society, 26:1421-1427, 2006. 
Table 1: Material properties used to validate the macroscale model.

\begin{tabular}{|l||l|l|l|l|l|l|l|}
\hline Material & $\begin{array}{l}\text { Average } \\
\text { Pore Size } \\
(\mathrm{nm})\end{array}$ & $\begin{array}{l}\text { Thickness } \\
(\mathrm{nm})\end{array}$ & $\begin{array}{l}\text { Effective } \\
\text { Fracture } \\
\text { Toughness } \\
(\mathrm{MPa} \sqrt{\mathrm{m}})\end{array}$ & $\begin{array}{l}\text { Porous } \\
\text { Young's } \\
\text { Modulus } \\
(\mathrm{GPa})\end{array}$ & $\begin{array}{l}\text { Residual } \\
\text { Stress } \\
(\mathrm{MPa})\end{array}$ & $\begin{array}{l}\text { Poisson's } \\
\text { Ratio }\end{array}$ & Porosity \\
\hline \hline pnc-Si & 28 & 20 & 0.98 & 128 & 57 & 0.22 & $7 \%$ \\
\hline NPN & 66 & 50 & 1.45 & 93 & 122 & 0.28 & $20 \%$ \\
\hline NPN & 55 & 50 & 1.41 & 108 & 127 & 0.28 & $14 \%$ \\
\hline
\end{tabular}


Table 2: Comparison of the fracture toughness values used in this publication. All values are generally subject to $20 \%$ uncertainty leaving room for small calibrations in the current work. ${ }^{a}$ Experimental spread from best-fit effective fracture toughnesses. ${ }^{b}$ Values indicate corrected experimental spread according to Picard et al. [32]. ${ }^{c}$ Average value and spread. ${ }^{d}$ Bulk values of crystalline $\mathrm{Si}_{3} \mathrm{~N}_{4}$ and $\mathrm{Si}$ are indicated for comparison to our amorphous thin film tests.

\begin{tabular}{|c|c|c|c|c|}
\hline Source & $\begin{array}{l}\text { Material } \\
\text { Morphology }\end{array}$ & Test Method & $\begin{array}{l}\operatorname{SiN}_{\mathrm{x}} \mathrm{K}_{\mathrm{Ic}} \\
(\mathrm{MPa} \sqrt{\mathrm{m}})\end{array}$ & $\begin{array}{l}\text { Si K } \mathrm{Ic}_{\mathrm{Ic}} \\
(\mathrm{MPa} \sqrt{\mathrm{m}})\end{array}$ \\
\hline $\begin{array}{l}\text { Current } \\
\text { Work }^{a}\end{array}$ & $\begin{array}{l}\text { Sputter } \\
\text { Deposition } \\
\text { (amorphous) }\end{array}$ & Bulge & $1.43 \pm 0.15$ & $0.98 \pm 0.01$ \\
\hline Matoy $[18]^{b}$ & $\begin{array}{l}\text { PECVD } \\
\text { (amorphous) }\end{array}$ & $\begin{array}{l}\text { Micro- } \\
\text { Cantilever }\end{array}$ & $1.3 \pm 0.1$ & - \\
\hline Di Maio [19 $]^{c, d}$ & $\begin{array}{l}\text { Bulk } \\
\text { Crystalline }\end{array}$ & $\begin{array}{l}\text { Micro- } \\
\text { Cantilever }\end{array}$ & - & $1.1 \pm 0.016$ \\
\hline Wong [33 $]^{c, d}$ & $\begin{array}{l}\text { Bulk } \\
\text { Crystalline }\end{array}$ & $\begin{array}{l}\text { Double } \\
\text { Cantilever } \\
\text { Beam }\end{array}$ & - & $0.94 \pm 0.03$ \\
\hline Wong [33] $]^{c, d}$ & $\begin{array}{l}\text { Bulk } \\
\text { Crystalline }\end{array}$ & $\begin{array}{l}\text { Four-Point } \\
\text { Bending }\end{array}$ & - & $0.90 \pm 0.11$ \\
\hline Wong $[33]^{c, d}$ & $\begin{array}{l}\text { Bulk } \\
\text { Crystalline }\end{array}$ & Microindentation & - & $1.00 \pm 0.08$ \\
\hline Michot $[34]^{c, d}$ & $\begin{array}{l}\text { Bulk } \\
\text { Crystalline }\end{array}$ & $\begin{array}{l}\text { Double } \\
\text { Cantilever } \\
\text { Beam } \\
\end{array}$ & - & $0.93 \pm 0.05$ \\
\hline Anstis $[35]^{d}$ & $\begin{array}{l}\text { Bulk } \\
\text { Crystalline }\end{array}$ & Double Torsion & $2.1 \pm 0.2$ & $0.8 \pm 0.2$ \\
\hline Anstis $[35]^{d}$ & $\begin{array}{l}\text { Bulk } \\
\text { Crystalline }\end{array}$ & $\begin{array}{l}\text { Double } \\
\text { Cantilever } \\
\text { Beam }\end{array}$ & $4.0 \pm 0.3$ & - \\
\hline
\end{tabular}




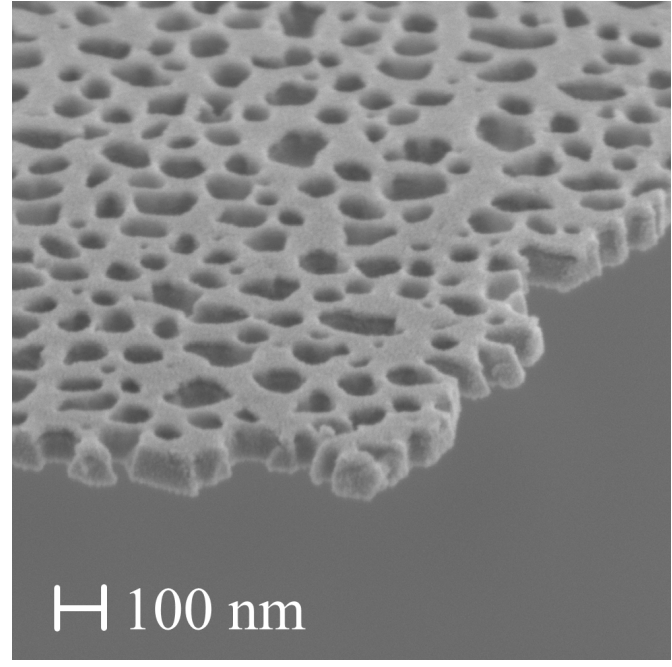

(a)

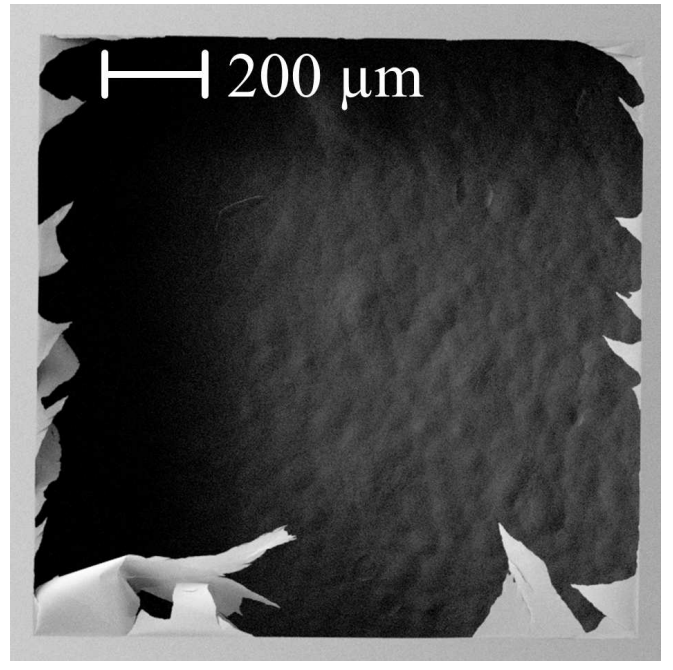

(b)

Figure 1: Scanning electron microscope images of the porous membranes. (a) A close up image of the pores that promote failure. (b) Stress concentrations from large bending moments at the edges of the square membranes produce sudden and catastrophic failure. 
Figure 2
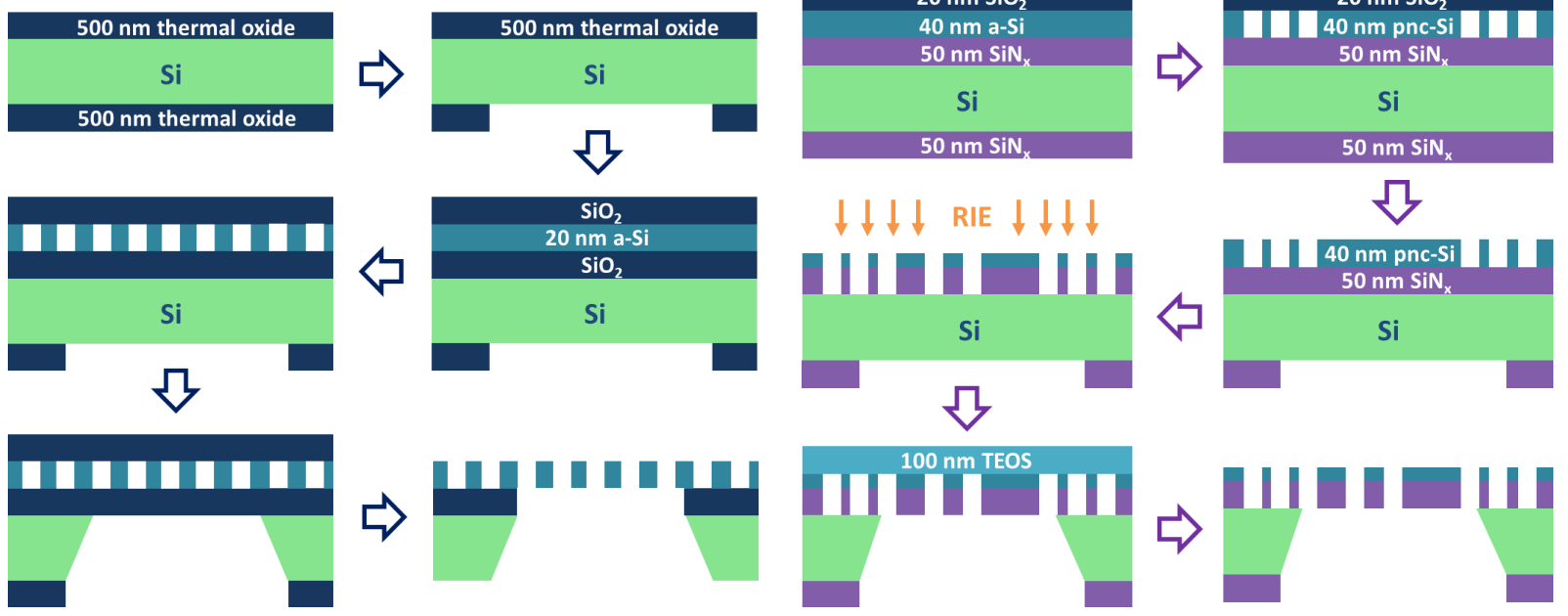

(a)
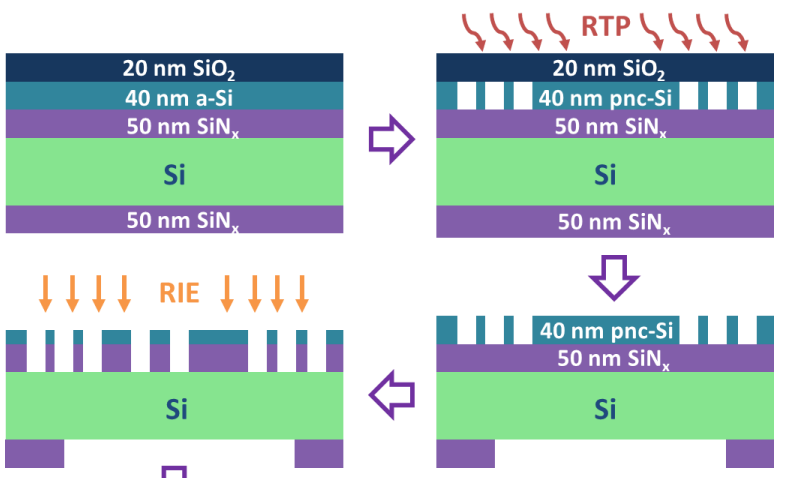

(b)

Figure 2: Process flows for construction of porous membranes made of (a) porous nanocrystalline silicon (pnc-Si) and (b) nanoporous nitride (NPN). Step-by-step processes are outlined in the text. 
Figure 3

\section{Interferometer}

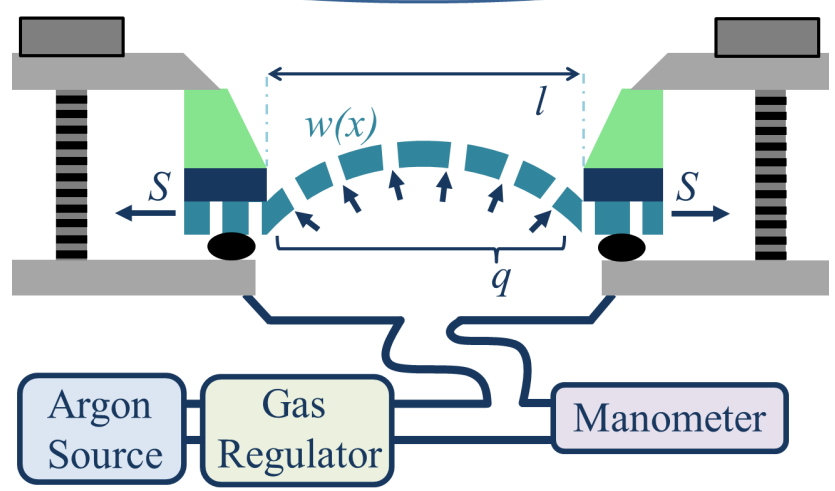

Figure 3: The initial two-dimensional case used to derive intrinsic stress in a bulge test scenario [14]. A membrane clamped at two edges is subjected to a uniform pressure, $q$. The membrane has length, $l$, thickness, $h$, and is subjected to a tensile force, $S$ as the membrane deforms under an applied load. The deflection of the membrane, $w$ is a function of its horizontal position, $x$. 


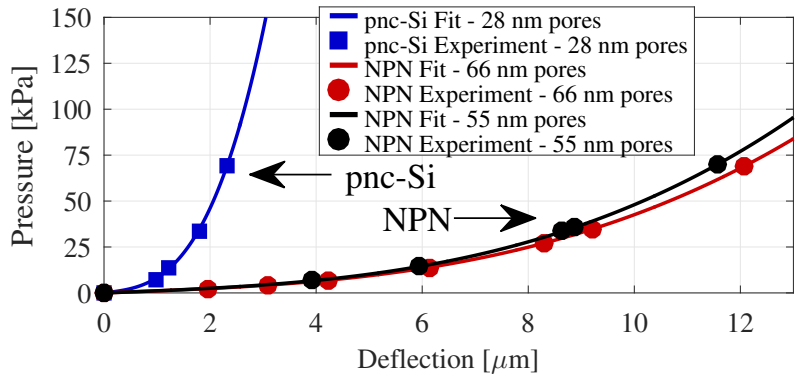

Figure 4: Experimental data obtained using the bulge test to measure porous effective Young's moduli and porous effective residual stresses of the membranes in situ. Measured parameters are outlined in Table 2. These membranes were not forced to failure. (Color online) 

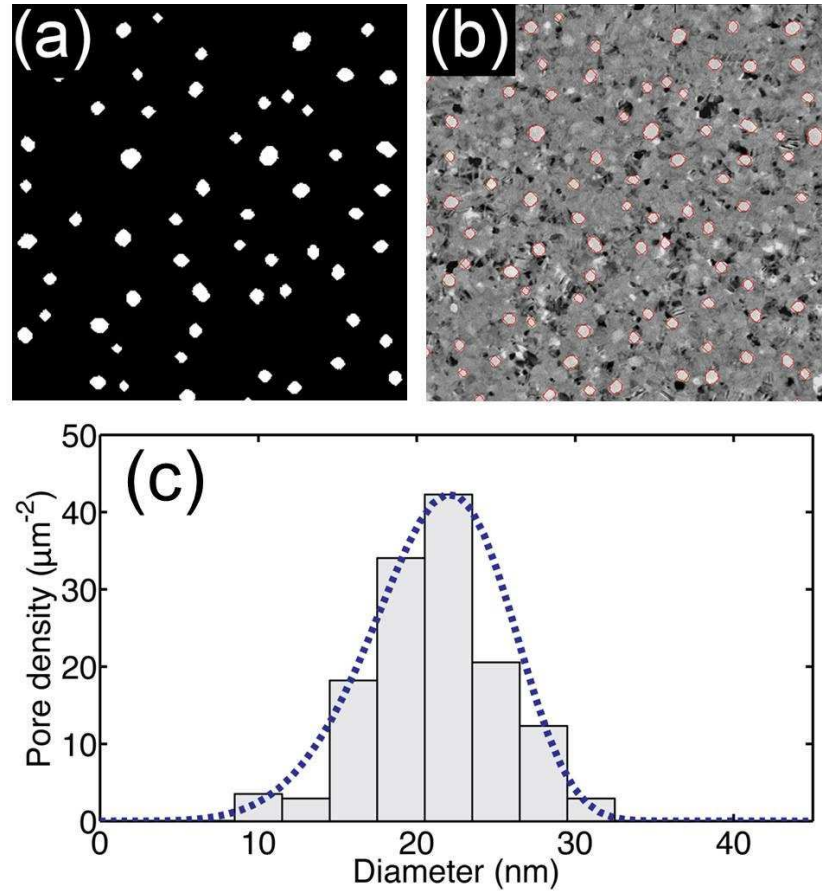

Figure 5: A representative pore size distribution on a pnc-Si membrane. To measure the porosity of the membranes in this work, (a) a threshold algorithm is applied to the TEM image to create a binary image, (b) pores are identified and (c) a size distribution calculated. A Weibull probability density fit is applied to the histogram (blue dashed line). (Color online) 


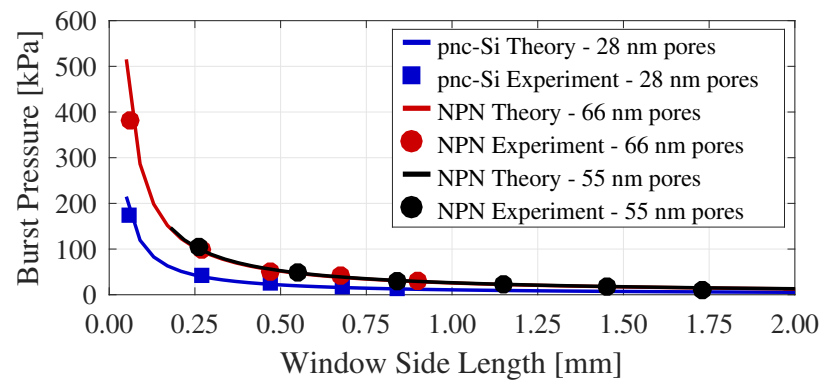

(a)

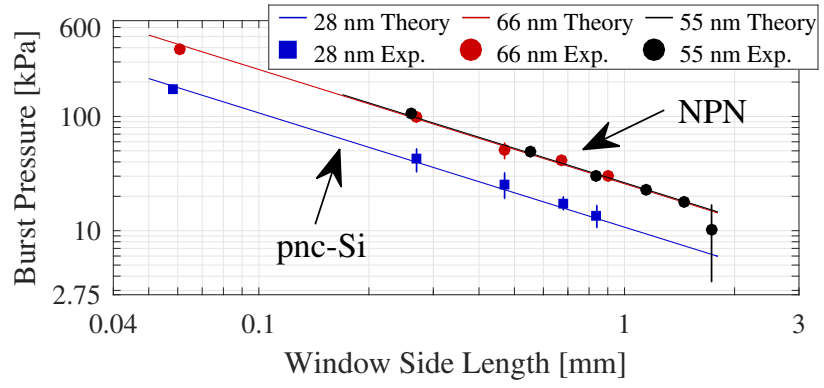

(b)

Figure 6: Predicted burst pressures vs. measurement for square membranes on (a) a linear scale and (b) a log-log scale. Error bars represent \pm one standard deviation of experimental results. (Color online) 


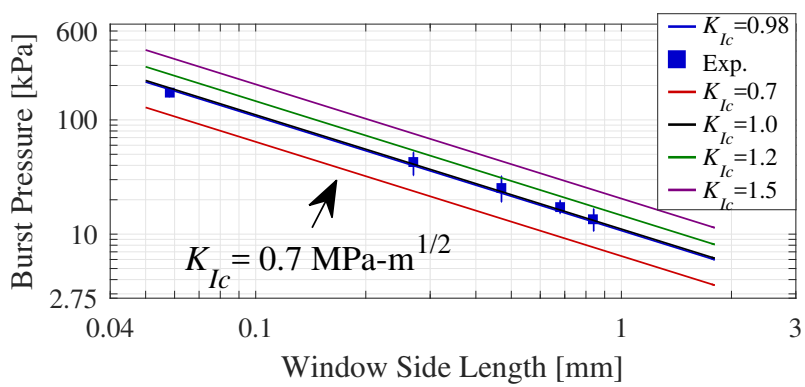

(a)

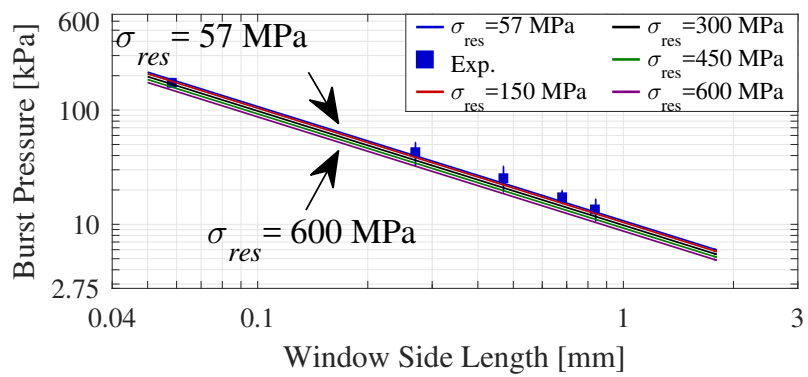

(c)

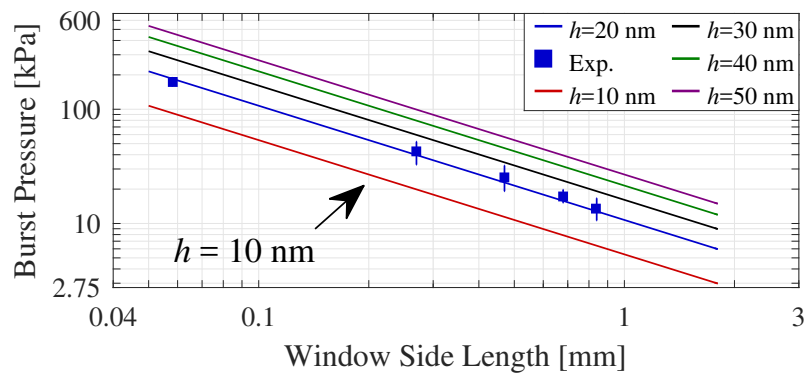

(e)

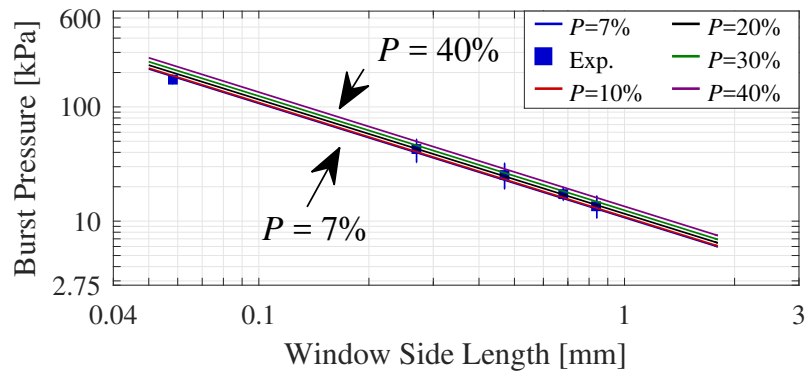

(b)

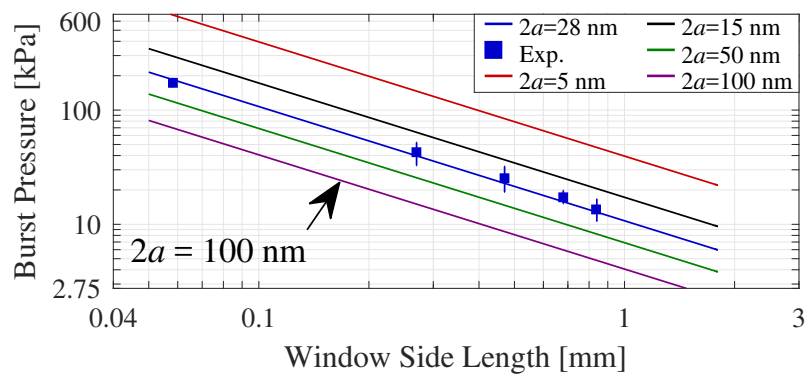

(d)

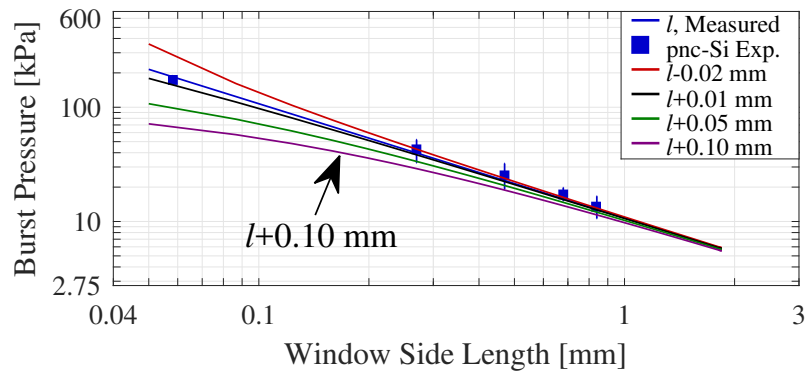

(f)

Figure 7: Exploring the parameter space using the numerical simulation. All blue lines are porous pnc-Si data from Figure 6 while additional trends represent changes in (a) fracture toughness, (b) porosity, (c) residual stress, (d) pore size, (e) membrane thickness, and (f) error in side length measurement. The burst pressure axis scale has been maintained across all plots for an intuitive comparison of parameter sensitivities. (Color online) 